\title{
VeloCity: Using Voice Assistants for Cyclists to Provide Traffic Reports
}

\author{
Gian-Luca Savino \\ University of Bremen \\ Bremen, Germany \\ University of St. Gallen \\ St. Gallen, Switzerland
}

\author{
Jessé Moraes Braga \\ University of Bremen \\ Bremen, Germany
}

\author{
Johannes Schöning \\ University of Bremen \\ Bremen, Germany \\ University of St. Gallen \\ St. Gallen, Switzerland
}

\begin{abstract}
Cycling is on the rise as a relevant alternative to car-based mobility and even though there are mobile applications specifically designed for cyclists to support this development, many still face unresolved challenges in terms of safe user interaction with complex data while riding. We present the design, development, and evaluation of VeloCity - an application for reporting traffic incidents and structures relevant to cyclists. In a case study, we compared its' three input methods (touch, in-app speech recognition, the voice assistant of the operating system) to evaluate which attributes make for safe interaction while cycling. We found that participants prefer to use the voice assistant over the other modalities as it was least distracting due to its hands- and eyes-free interaction design. Furthermore, they chose short commands over conversational phrases. Based on our results, we present five guidelines for designing voice user interfaces for cyclists and argue for moving away from touch-based interfaces in this domain, which still make up most of the applied interaction techniques today.
\end{abstract}

\section{CCS CONCEPTS}

- Human-centered computing $\rightarrow$ Interaction techniques; Usability testing; User studies; Mobile devices.

\section{KEYWORDS}

mobile interaction, cycling, voice interface, volunteered geographical information

\section{ACM Reference Format:}

Gian-Luca Savino, Jessé Moraes Braga, and Johannes Schöning. 2021. VeloCity: Using Voice Assistants for Cyclists to Provide Traffic Reports. In Proceedings of the 29th ACM International Conference on Multimedia (MM '21), October 20-24, 2021, Virtual Event, China. ACM, New York, NY, USA, 10 pages. https://doi.org/10.1145/3474085.3475509

\section{INTRODUCTION}

Bicycles are becoming an increasingly important means of transport in many cities as they complement or replace the car or public transport as a viable option [35, 36, 49]. Cities worldwide already

Permission to make digital or hard copies of all or part of this work for personal or classroom use is granted without fee provided that copies are not made or distributed for profit or commercial advantage and that copies bear this notice and the full citation on the first page. Copyrights for components of this work owned by others than the author(s) must be honored. Abstracting with credit is permitted. To copy otherwise, or republish, to post on servers or to redistribute to lists, requires prior specific permission and/or a fee. Request permissions from permissions@acm.org.

MM '21, October 20-24, 2021, Virtual Event, China

(C) 2021 Copyright held by the owner/author(s). Publication rights licensed to ACM ACM ISBN 978-1-4503-8651-7/21/10 .. \$15.00

https://doi.org/10.1145/3474085.3475509 have dedicated cycling infrastructure and more cities are following to implement car-free areas or so-called cycle highways [29, 45]. As the number of cyclists rises and cycling becomes one of the main transportation methods for daily commutes, cyclists need and use navigation applications more often. In the first half of the year 2020, Google Maps saw a worldwide increase of $69 \%$ in routing requests for bicycles [22]. Not only specific cycling modes are implemented within existing navigation applications (e.g. Google Maps or Strava [43]), but also apps or new devices (e.g. Komoot [21], Beeline [5], SmartHalo [41]) are developed, which aim to improve the overall cycling experience and provide navigation instructions tailored to cyclists.

However, navigation apps for cyclists still lack information on real-time traffic, incidents and obstacles. Car drivers can get such information through various services which report traffic jams, closed lanes or roads, construction sites, dangerous objects on the road or other incidents. Most of the time such information is embedded directly within the navigation app. For example, the navigation app Waze [47] uses volunteered geographical information (VGI) [16], which is mainly provided by its users to improve their routing and inform drivers about traffic-related incidents. This information can also be shared with public authorities (as Waze does in some cases) to see where road infrastructure needs improvement. We see an emergent need for similar services for cyclists, to improve routing and infrastructure in the future. However, interacting with such a service while riding could pose security risks as most cycling applications are operated using touch interaction. They either require a person to hold a smartphone or strap it to their handlebar similar to how dedicated devices are usually fixed to the bicycle's handlebar $[5,41]$. Therefore, we explore the voice as a possible interaction technique that is suited to safely report traffic incidents and obstacles while cycling. While audio has been proven to work well for cyclists to receive information [13, 28, 38] (e.g. turn-by-turn instructions), there is only limited work on voice as a method to input data while cycling. Thus, these efforts do not only improve the safety of cyclists but also enable them to interact with more complex data than before. Consequently, in this paper we investigate the following research question:

RQ1: Which inputs methods are suited for cyclists to safely report real-time traffic, incidents, and obstacles?

In a pre-study, we used a user-centred design process to identify participants needs and behaviours, which informed the design of our application as well as the comparison of viable interaction techniques. Based on our findings we developed an app called VeloCity that allows users to report real-time traffic information for cyclists. In a user study $(\mathrm{n}=21)$ we compared three interaction techniques 
(touch, in-app speech recognition, and using the operating systems' voice assistant), that enable cyclists to provide this information during live traffic.

We found that voice-based interaction was preferred over touch to interact with the reporting app VeloCity. Still, both touch and speech recognition were similarly distracting for participants, while only the voice assistant allowed them to pay more attention to traffic. This was due to the hands- and eyes-free interaction that the voice assistant offered, which even novices, who have not been using voice assistants before, quickly learned to take advantage of. There was no significant difference between the usability of all three techniques. Based on these results we formulate five guidelines for designing voice user interfaces (VUIs) for cyclists to provide traffic reports. Consequently, this paper contributes (1) the first, to our knowledge, study on viable interaction techniques to report live traffic data while cycling; (2) the use of voice assistants specifically for cycling tasks to enable safe, hands- and eyes-free interaction.

\section{RELATED WORK}

To identify interaction techniques suitable for reporting live traffic incidents while cycling, we review previous work on cycling in $\mathrm{HCI}$ and interaction in motion.

\subsection{HCI \& Cycling}

Research on cycling in HCI looks at two perspectives: Input from a user to a system or output from the system to the user. Especially in the domain of navigation, there is a lot of related work on how to inform the user through different kinds of output methods. Pielot and Poppinga et al. [32, 33] for example used vibrating handlebars in a navigation setting. Through the vibration of the left and right handle, they communicate the direction to a destination in an as-the-crow-flies manner. Albrecht et al. [2] used spatial audio and Savino et al. [39] a visual display to convey similar information. Another approach to tactile information is Vibrobelt. The belt, worn around the waist, gives waypoint, distance and endpoint information using directional tactile cues and was able to successfully navigate participants over an unfamiliar route [42] For a pure turn-by-turn style approach, Huxtable et al. [18] present a tactile interface for wayfinding devices designed for cyclists. It is made up of two wristbands that vibrate to signal left and right turns. In terms of improved visual map approaches, as many navigation applications use them, Dancu et al. [12] investigate to move this visualisation into the environment through a map which is projected in front of the bicycle with the goal to make cyclists more attentive to their environments. All of these systems do not only enable cyclists to interact with information while cycling, most of them also aim towards making this interaction as safe as possible by directing the user's visual attention away from the mobile device. Because, not only are cyclists victims of bicycle-motor vehicle crashes, single-bicycle crashes (i.e. a fall or obstacle collision) are as well a serious problem that needs to be kept in mind when designing interactive systems for cyclists [40]. About half of all single-bicycle crashes are related to infrastructure such as collision with an obstacle, riding off the road or skidding due to slippery surfaces [40]. To counter bicycle-motor vehicle crashes, Carton [10] presents a solution to improve the visibility of the rider's hand gestures. LED's attached to gloves will illuminate, depending on the gesture the gloves recognised, and thus amplify the gesture, especially at night times. An approach to warn cyclists themselves was tested by Matviienko et al. [27]. They used multimodal warning signals for child cyclists and found that reaction times could be reduced when using a combination of tactile, visual, and auditory feedback over unimodal warnings. After presenting systems that make the output available to users and care for their safety, we now look at systems that actively enable cyclists to interact not only with the information mobile systems provide but also with the systems themselves through an appropriate interface.

\subsection{Interaction in Motion}

Interacting with mobile devices on a bicycle can be very dangerous [14]. Still, people interact with these systems while in motion, including cycling [25]. To better understand the reasons and consequences behind this phenomenon, Marshall et al. [25] classify interactions in motion with regards to the locomotion's inhibition on interaction and the interactions related to the locomotion. Within this taxonomy, cycling is classified as a strongly constrained activity as hands and feet are occupied with operating a vehicle. And even though mobile devices are increasingly portable, most systems are not designed for meaningful two-way interaction whilst moving [26]. Because except for navigation applications, most interactions on the bicycle are unrelated to locomotion. The constraints on hand (and foot) interaction, which cycling creates, are especially relevant at points when one may wish to manoeuvre. Related work takes on this problem from many different angles. Especially when you are confronted with an obstacle, fast manoeuvres can be necessary. Thus, touch interaction seems unfitted for the task of logging these obstacles while riding. While using large user interface elements (like buttons) has been shown to work for pedestrians while walking [19], studies have found that cyclists prefer techniques where they do not have to take their hands off the handlebar [13]. Voice-based interactions, therefore, seem like a good fit. However, they come with the disadvantage of potentially being masked by traffic noise or the necessity to wear headphones, which in return mask traffic noise and are thus a potential safety hazard [38].

Aside from touch and voice as input methods, related work has explored a variety of ways how cyclists can interact with mobile devices. Dancu et al. [13] explore gesture-based input. Through hand gestures, cyclists can control a turn indicator that is mounted to the bicycle. This is an example of a "Tailored Solution" regarding the taxonomy by Marshall et al., as the turn gesture (stretching one arm outwards to signal a turn) is encouraged by the locomotion itself. Vechev et al. [46] investigated an interface that allows tapping different areas on the user's body to interact with a mobile system while cycling. This, as well as the above strategy, however, require removing one's hands from the handlebar. In response to this disadvantage, Woźniak et al. [48] created custom handlebar controllers. These feature buttons as well as moving parts to interact with the most common smartphone features like accepting a phone call or play/pause music.

To expand the research on existing interaction techniques, we explore leveraging the possibilities of built-in voice assistants to report live traffic incidents for cyclists. Voice assistants are being 
successfully used in other domains [3] to interact with multimedia data and are already widely available and easy to connect to existing systems.

\section{PRE-STUDY}

The goal of the pre-study was to build an in-depth understanding of what interaction techniques people naturally use while cycling. In this study, we used a contextual inquiry [6] approach (similar to [44]). This allowed us to observe users in a real-life situation, and learn how they would use an application to report live traffic information for cyclists. Therefore, an experimenter together with a participant embarked on a bike ride and introduced imaginary obstacles along the road which the participant should log using an imaginary application. We made sure all participants were experienced bike riders and explained that they should only interact in ways they felt safe and comfortable with. Since we focused on a smartphone application we mounted a smartphone that was turned off to the handlebar. The goal of this method was to observe participants in-situ to learn what kind of incidents/obstacles they would report and what interaction techniques they would use doing so.

\subsection{Participants}

We used convenience sampling to recruit six participants to take part in the pre-study. All participants were equipped with an omnidirectional lapel microphone connected to a smartphone that was used to record them thinking aloud [30]. All of the participants reported riding bicycles on a casual (at least once a week) to regular (3 or more times a week) basis. Everyone took part voluntarily and was not compensated for their participation.

\subsection{Procedure}

The entire experiment lasted for 30 minutes. Participants were familiarised with the experiment which asked them to use an imaginary mobile application for reporting live traffic incidents while riding a bicycle in real-life conditions (i.e. sharing traffic with cars, other bicycles, and e-scooters, dodging construction sites, roadblocks, pedestrians, etc.). They were asked to think aloud during the whole experiment. During the ride, participants encountered imaginary obstacles introduced by the experimenter which were inspired by the ADFC "Mängelmelder", a web platform where citizens can report obstacles and incidents for cyclists [1]. In addition to those, real-life obstacles were encountered which participants should also report to the app. They imagined their own version of the application including how it looked and how the interaction worked. As such, we could observe the challenges of the reporting task and the constraints they dealt with while cycling. Participants were offered to ride the same route a second time, which allowed them to get used to the demanding conditions and think more creatively. In the end, we used an unstructured interview to ask them about specific events, their reasons for the chosen interaction technique, and their general opinion on a traffic reporting application for cyclists.

\subsection{Results}

Once participants were familiar with the task, they felt comfortable making suggestions and interacting with the app in a way that felt natural to them. They changed from stopping to interact via touch to using voice commands and voice assistants while in motion. Regarding voice commands, they mainly used short words to describe the obstacle and mentioned that "[...] it would be nice if the app could translate what you say into categories" (participant 6) or that "it would be just easier to say keywords that are already a category in the app, so the app would understand me" (participant 6). Another participant commented that he would prefer to communicate with short sentences, but the assistant should understand full sentences. An example phrase for a voice assistant was "Hey bicycle app, please log shattered glass to my position" (participant 3). These insights helped us to develop and design the VeloCity application as well as the main study.

\section{VELOCITY APPLICATION}

VeloCity was developed in Swift 5 and is compatible with iOS 13.2 and newer. The application consists of four main sections: (1) The map-view features a map that shows the users current location. This view features an action button that, depending on the condition, is used to report incidents via touch or speech recognition (see figure $1 \mathrm{~b}$ and $1 \mathrm{e}$ ). The live location feature and the map, although not necessary for the experiment, were included to give a realistic look and feel of the app, as this was how participants imagined the app to look like in the pre-study. Additionally, a recording button on the screen is used to mark the start and end timestamps in the application logs during the experiment. This way we were able to differentiate between trial runs and the actual conditions. (2) The incident list features all incidents which have been reported during a session (see figure 1i). It serves as a visualisation of the result of each condition and allows participants to correct their inputs afterwards. This was used to check how many times participants realised they made a mistake and wanted to correct it after a session. (3) The survey view contains the surveys for the main study. These can be filled in right in the app. (4) In the settings participants can input their participant-ID, change the input method and the language of the application (see figure 1a). The input method could be changed between touch input and speech recognition. This changes the action button in the map view which then triggers the corresponding input when tapped. The experiment was conducted in two languages depending on the participant's preferences. The language could be switched between German and English in the settings.

\subsection{Input Methods}

Based on the pre-study we identified three input methods that are relevant for cyclists when reporting live traffic information: touch, in-app speech recognition, and the built-in voice assistant (Siri). These were implemented into the VeloCity application. Touch is state of the art in other comparable applications such as Waze and functions as a baseline. Speech recognition and the built-in voice assistant were proposed by participants in the pre-study as a successful technique to interact while cycling. Since it was not clear which one they would favour, and how exactly they would perform, we implemented and tested them in our main study.

4.1.1 Touch. Figure $1 \mathrm{~b}-1 \mathrm{~d}$ shows the user story for adding an incident using touch. The user taps the action button, selects a category, and is presented with the confirmation that the incident was added. 


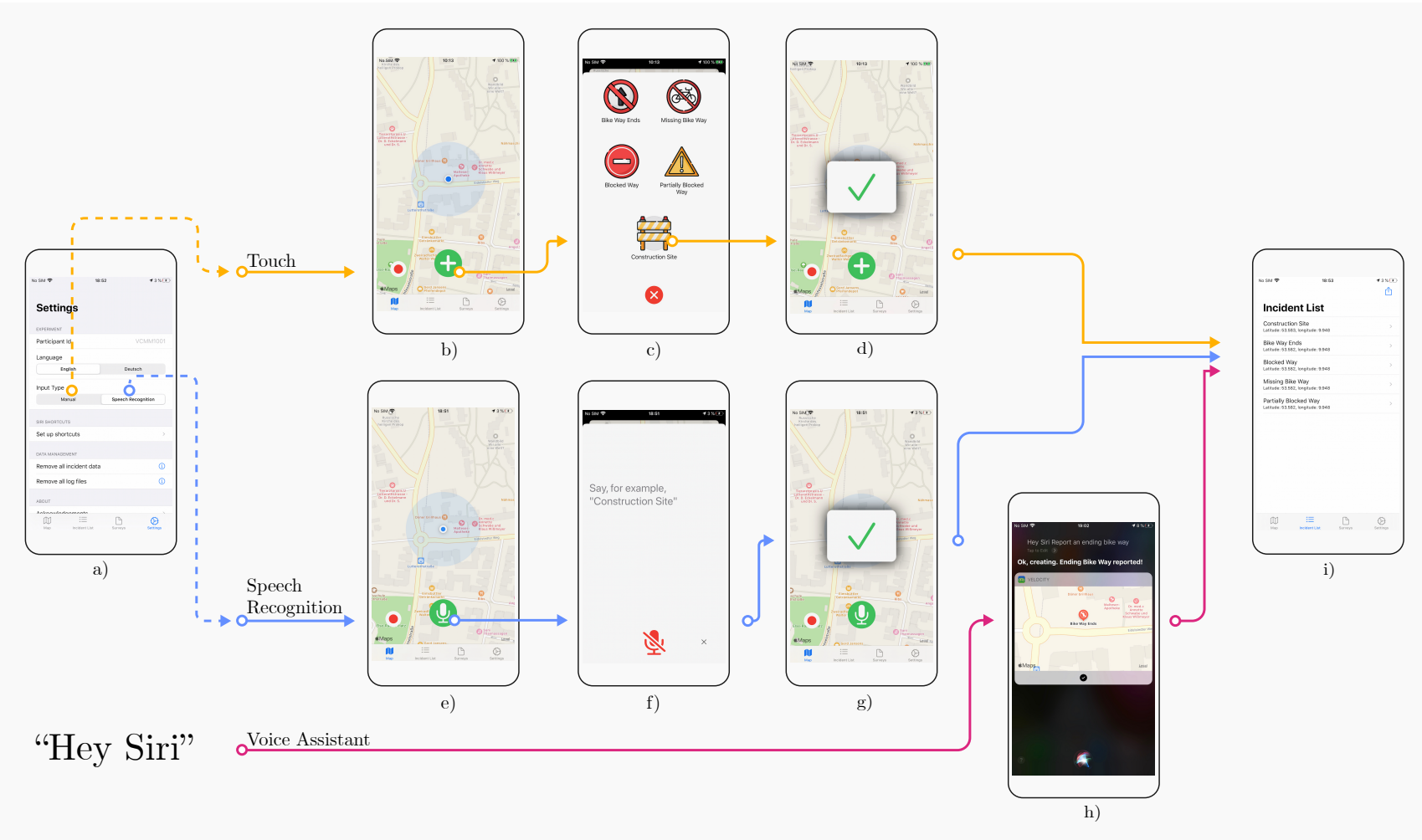

Figure 1: User Story of the VeloCity application. Depending on the condition, the participant switched on either "Manual" (touch) or "Speech Recognition" in the Settings. The green action button then changes to a plus sign (touch) or a microphone (speech recognition). The participant can then report an obstacle as shown above and gets visual as well as auditory feedback (only in the speech recognition condition) after a successful report. The activation phrase "Hey Siri" can be used from anywhere, even when the device is locked. Eventually all reports are saved to the Incident List.

The look and feel of the touch condition were inspired by Waze. We used a similar layout using expressive icons to present the obstacles that can be reported. This was also in line with how participants imagined the application in the pre-study. If a participant accidentally pressed the action button, the incident view (figure 1c) could be dismissed by pulling it down.

4.1.2 Speech Recognition. Reporting an incident via speech recognition is similar to the touch input (see figure 1e-1g). After the user taps the action button, the app will play a sound to cue the user that it is ready to record. Similarly, the app will play sounds when the user cancels or dismisses the view (by pulling down on it), as well as when the speech recognition is able to recognise a voice command. The latter case also dismisses the voice recognition view and displays a confirmation on the map view (see figure $1 \mathrm{~g}$ ). To make for a robust implementation, we use iOS's built-in speech recogniser in combination with the fuzzywuzzy_swift library [24] which uses Levenshtein distance to find the most similar incident category to the spoken input. If the input roughly matches one of the categories the app finds a match and saves the incident. This meant that the speech recognition would understand even short phrases like "construction" and map them to the appropriate category (in this case "construction site"). This resulted in very few cases where the speech recognition did not recognise the correct category.

4.1.3 Voice Assistant (Siri). For the voice assistant condition, we implemented six shortcuts that can be added to Siri - one for each incident category and a generic one, that can add any category. Shortcuts offer the possibility to add custom commands to Siri and connect them with your own application. These shortcuts must be set up once but can then be used from anywhere on the device. Thus, the app could be running in the background, or even be completely out of iOS' memory. In our experiment, we instructed participants to use the "Hey Siri" command in combination with the custom shortcuts such as "Hey Siri, report an ending bikeway" (see figure 1h). As this example highlights, in contrast to our speech recognition, Siri needed a full sentence to understand the command. This was to make it as conversational as possible.

\section{STUDY}

Using the VeloCity application, the study addresses the research question (RQ1: Which input methods are suited for cyclists to report traffic incidents?) by looking into which of the investigated input methods participants prefer and how they perform in regards to attention and usability. 


\subsection{Study Design}

In order to reach a diverse sample and control for hard to control factors like weather, traffic and the type of bicycle, the study was conducted remotely and under lab settings at a desk meaning that participants did not actually ride a bike in traffic. This was done to minimised safety risks as participants would use real interaction techniques designed by us, which could harm them when used in real traffic and without sufficient training.

Participants installed the VeloCity application on their own iOS devices and were sent three videos of the experimenter riding a bicycle through an urban environment, one for each of the three conditions. Using a within-subjects design each participant went through each of the three conditions using the three different input methods respectively. The order of the conditions was randomised using a Latin square design, keeping the order of the videos and permuting the input methods.

\subsection{Participants}

We used convenience sampling to recruited 21 participants from different backgrounds such as five different countries (Brazil, Canada, Belgium, Denmark, Germany) and six nationalities (Russian, Turkish, Brazilian, Irish, Maltese, German). Of our participants, 52\% identify as female, $43 \%$ identify as male, and $5 \%$ identify as diverse. Moreover, $19 \%$ are aged 18 to $24,48 \% 25$ to $30,24 \% 31$ to 40 , and $9 \%$ over 40 . When it comes to participants' cycling habits, most of them cycle on a weekly basis (38\% of respondents answered with "1-2 days a week" or "3 to 7 days a week") and have their own bicycle. When asked about the reasons for cycling we found that cycling is still often seen as a hobby, but health and environment-related reasons also play a role alongside. With regards to the technology used while cycling, apps are more popular among our sample than dedicated cycling gadgets (like Beeline [5]). 8 participants use smartphone apps, 1 participant uses a sports watch, 1 participant uses a smartwatch and 1 participant a bike computer. When asked how they carry their smartphone while cycling, 8 replied that they carry it in their pockets and take it out, when needed. When it comes to voice assistants like Siri, Alexa or Google Home, about half of our sample (10 out of 21) reported using some kind of assistants, mainly to automate simple tasks, such as setting an alarm or timer, play music, or check the weather.

\subsection{Procedure}

The entire experiment lasted for about one hour and was video recorded for later analysis. Previous to the session, participants were sent information material on how to install and set up the application. The experiment started with a greeting and a brief explanation of the study. All participants were informed that their participation is voluntary and that they can choose to end the experiment at any given time. The experimenter then ran through a checklist, checking the in-app settings and settings of the operating system (e.g. whether "Hey Siri" was activated). A brief explanation of the app was given, showing the user an introductory video of a sample reporting task. The different incident categories were explained and how to report them correctly using the different input methods. For the main task, participants were asked to set up the respective input method and place their phone on a flat surface

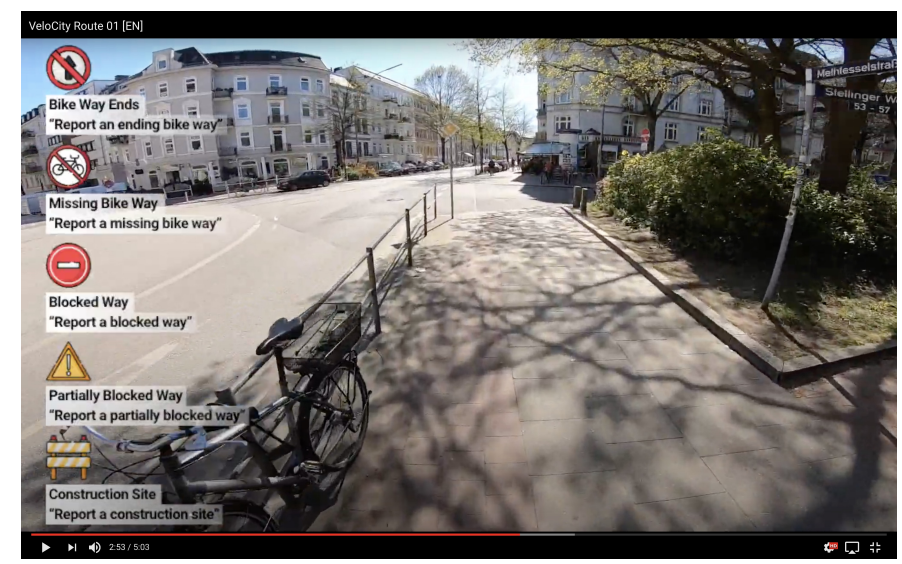

Figure 2: Screenshot of one of the videos participants saw during the experiment. In order to remember the five different obstacle categories and the respective commands to report them, they were always included in the video on the left side of the screen.

in front of them. They were asked to not hold the phone in their hands as we wanted to imitate the phone being strapped to the handlebar. Before each condition participants were given time for a dedicated training phase and were able to ask questions until they felt comfortable using the input method. They were then asked to open a link to a video of an experimenter driving a bicycle through an urban environment (see figure 2). In order to remember the different incident categories, they were displayed in the video at all times. While watching the video, participants were asked to report any of the five obstacles they noticed. Each video was 5 minutes long and participants were asked to report all incidents without stopping it. After each video participants answered a questionnaire asking them about their experience with the just used input method and its usability. Finally, a short, unstructured interview with participants has conducted at the end of the study.

\subsection{Measures}

Using a mixed-methods approach, we collected data from the application logs, the experiments' recordings, the surveys and the interviews (which were loosely transcribed for the analysis). The app itself logs all user interactions and settings in a text file as soon as it is opened for the first time. This log data includes language used to complete the conditions, timestamps of when a reported obstacle was added, edited, or deleted, etc. The main measures that are taken to answer our research question are:

Personal Preference: We compared identical questions from the questionnaire among all three input methods, in order to determine which one users liked best.

Attention: Participant's visual attention is measured by counting how many times they looked at the device's screen. Video recordings of the call were used for the analysis.

System Usability Scale: We use the System Usability Scale (SUS) [9], to assess which of the three input methods was better rated by users, in regards to usability. 


\subsection{Results}

All 21 participants were able to complete the experiment. The data presented in this section were statistically analysed in $\mathrm{R}$ using the following methods: The Shapiro test plus visual analysis of histograms were used to determine normal and not normal distributions. Comparisons between groups were done using the KruskalWallis test with Wilcoxon tests for pairwise comparisons for not normally distributed data. For normally distributed data we used the Student's t-test. All tests use an alpha of 0.05. Effect sizes were calculated according to Robertson and Kaptein [37].

5.5.1 Personal Preference. We used surveys after each condition to ask participants about different aspects of the input method they just used. They answered using a five-point Likert scale. We analysed the answers to each of the statements using a Kruskal-Wallis test and a Wilcoxon-Pratt test for pairwise comparisons. We also provide effect sizes for all of those results. For a complete overview of the statements, answers and results please refer to table 1. Overall we find that participants rate both speech recognition (median: 4, p-value: 0.009 ) and voice assistant (median: 4, p-value: 0.006) more useful than touch (median: 3). Furthermore, we find participants could better keep their eyes on the road in the speech recognition (median: 4, p-value: 0.0009) and voice assistant (median: 5, p-value: 0.0001 ) condition than in the touch condition (median: 2) (see table 1). There is no significant difference between speech recognition and voice assistant ( $p$-value: 0.062$)$ but a medium effect size $(-0.294)$ suggests that we might find a difference with a bigger sample. Regarding the question of whether participants felt distracted by the input method, we find significant differences between all conditions. Touch was rated more distracting than speech recognition (p-value: 0.008$)$ and more distracting than the voice assistant ( $p$ value: 0.0153$)$. The voice assistant was rated less distracting than speech recognition (p-value: 0.002). Thus, participants were most distracted by their phone in the touch condition (median: 4). The second most distracting input method was speech recognition (median: 3) and the least distracting the voice assistant (median: 2) (see table 1). Participants had the option to correct wrong inputs after each video. We find that participants in total reported having corrected 5 incident reports created via touch, 12 via speech recognition and 15 via voice assistant. Thus touch seemed to be the most accurate input method.

5.5.2 Attention. We analyse visual attention to investigate if participants actually did not look at the screen if they did not have to. Siri can be used completely without looking at the device, which can be crucial for cyclists whose visual attention should be on the environment and the traffic at all times. From related work, we learn that such interfaces are more and more used in situations where complex tasks are done eyes-free [15]. Additionally, our intuition tells us that participants should have to look less or not at all at the device to report an obstacle using the voice assistant. To test this assumption we formulate the following hypothesis.

H1: Participants need fewer looks per interaction using the voice assistant compared to the other techniques.

To test our hypothesis, we used a classification method similar to Bah et al. [4]. We classified participants' looks at the screen when logging a traffic incident. We found that on average participants using touch needed 1.79 looks per interaction, with speech recognition they needed 1.71 and using the voice assistant 0.96 looks per interaction. To check whether these differences are significant, we used a Kruskal-Wallis test with posthoc pairwise Wilcoxon rank sum tests.

The Kruskal-Wallis test found a significant difference between the three conditions ( $p$-value $=0.0003$ ). Pairwise Wilcoxon tests showed that whereas touch and speech recognition $(\mathrm{W}=231.5$, $\mathrm{p}$-value $=0.7912)$ did not differ significantly from one another, touch and voice assistant $(\mathrm{W}=361.5$, $\mathrm{p}$-value $=0.0004)$ as well as speech recognition and voice assistant $(\mathrm{W}=355$, $\mathrm{p}$-value $=0.0007)$ do differ significantly. Thus we conclude that participants needed fewer looks per interaction with the voice assistance compared to touch and speech recognition respectively, which confirms our hypothesis.

5.5.3 Usability. We used the SUS to identify differences between the conditions regarding usability. A Kruskal-Wallis test found no statistical difference between the conditions $\left(X^{2}=2,5261, \mathrm{df}=\right.$ 2 , $\mathrm{p}$-value $=0,2828)$. However we found medium effect sizes for the comparison between touch and speech recognition inputs $(\mathrm{r}$ $=-0,2177683)$, as well as touch and voice assistant inputs $(\mathrm{r}=$ $-0,2362693)$, and small effect size for speech recognition and voice assistant inputs $(r=0,04042902)$. Therefore significant differences might be found between the first two mentioned comparisons with a larger sample [37].

\section{GUIDELINES FOR USING VOICE INTERACTION FOR CYCLISTS}

Many navigation apps are designed for multiple transport modalities but do not adapt their interaction techniques accordingly. This leads to increased risk for cyclists who use these services and applications. We thus see the need for more user-specific solutions for cyclists [48] which take their movement and inhibition on interaction with hands and feet into account. Therefore, in this section, we will discuss five guidelines we identified in our study when it comes to designing VUIs for cyclists to provide traffic reports.

\subsection{Use Hands and Eyes-Free Interaction}

According to the "four strategies for mobile interaction" by Marshall et al. [25] VeloCity falls into the "Danger Zone". This is because reporting obstacles while riding a bicycle is weakly related to the locomotion itself and cycling has a high inhibition on interaction with hands and feet being occupied. One way to design for the "Danger Zone" is to choose modalities that are easily accessible whilst moving [25]. Even though our experiment was stationary, the most preferred interaction technique was the voice assistant which can be used hands and eyes-free and is thus very easily accessible whilst moving which supports their design recommendation. Participants commented on this matter also showing their immersion in the task: "With Siri, it was good that you didn't have to touch the phone. You have to have your hands on the handlebar after all" (participant 3). To the question of which input method they preferred one participant answered: "Overall Siri. The reason is that you do not need to look at the phone" (participant 6). Participants had to look on average almost twice as much at the device to report an obstacle using touch or speech recognition compared to using the voice assistant 


\begin{tabular}{|c|c|c|c|c|c|c|c|c|c|c|c|c|c|c|c|}
\hline \multirow[t]{2}{*}{ Question } & \multicolumn{3}{|c|}{ Median Answer } & \multicolumn{3}{|c|}{ Kruskal-Wallis } & \multicolumn{3}{|c|}{ Wilcoxon-Pratt Z-Value } & \multicolumn{3}{|c|}{ Wilcoxon-Pratt $\mathrm{p}$-Value } & \multicolumn{3}{|c|}{ Effect Size } \\
\hline & TO & SR & VA & $\mathrm{X} 2$ & Df & $\mathrm{P}$-value & TO-SR & TO-VA & SR-VA & TO-SR & TO-VA & SR-VA & TO-SR & TO-VA & SR-VA \\
\hline $\begin{array}{l}\text { Rate this input method based on its usefulness when riding a } \\
\text { bicycle - very useful (5) to not useful at all (1) }\end{array}$ & 3 & 4 & 4 & 7.0965 & 2 & $\begin{array}{c}0.029 \\
*\end{array}$ & -2.464 & -2.7 & -0.288 & $\begin{array}{c}0.009 \\
* *\end{array}$ & $\begin{array}{c}0.006 \\
* *\end{array}$ & 0.792 & -0.380 & -0.416 & -0.044 \\
\hline $\begin{array}{l}\text { I could keep my eyes on the road most of the time - strongly } \\
\text { agree (5) to strongly disagree (1) }\end{array}$ & 2 & 4 & 5 & 18.339 & 2 & $\begin{array}{c}0.0001 \\
* * *\end{array}$ & -3.095 & -3.578 & -1.909 & $\begin{array}{c}0.0009 \\
* * *\end{array}$ & $\begin{array}{c}0.0001 \\
* * *\end{array}$ & 0.062 & -0.477 & -0.552 & -0.294 \\
\hline $\begin{array}{l}\text { I felt like the phone distracted me - strongly agree (5) to } \\
\text { strongly disagree (1) }\end{array}$ & 4 & 3 & 2 & 25.612 & 2 & $\underset{* *}{0.0027}$ & 2.626 & 3.853 & 3.014 & $\begin{array}{c}0.008 \\
* *\end{array}$ & $\begin{array}{l}0.0153 \\
*\end{array}$ & 0.002 & 0.405 & 0.594 & 0.465 \\
\hline $\begin{array}{l}\text { How do you classify logging incidents to the VeloCity App } \\
\text { with said input type, while watching the recording of someone } \\
\text { else riding a bicycle? - very easy (5) to very hard (1) }\end{array}$ & 4 & 4 & 4 & 1.997 & 2 & 0.368 & -1.315 & -1.056 & 0.088 & 0.213 & 0.32 & 0.9500 & -0.203 & -0.163 & 0.013 \\
\hline $\begin{array}{l}\text { The haptic feedback helped me notice I had tapped a button } \\
\text { - strongly agree (5) to strongly disagree (1) }\end{array}$ & 3 & 4 & NA & NA & NA & NA & -0.892 & NA & NA & 0.388 & NA & NA & -0.137 & NA & NA \\
\hline $\begin{array}{l}\text { The feature is accurate - strongly agree (5) to strongly } \\
\text { disagree (1) }\end{array}$ & NA & 5 & 5 & NA & NA & NA & NA & NA & 0.858 & NA & NA & 0.5 & NA & NA & 0.132 \\
\hline $\begin{array}{l}\text { I felt like the feature really understood what I said - strongly } \\
\text { agree (5) to strongly disagree (1) }\end{array}$ & NA & 5 & 5 & NA & NA & NA & NA & NA & 0.972 & NA & NA & 0.433 & NA & NA & 0.15 \\
\hline $\begin{array}{l}\text { The feature was able to classify incidents correctly most of } \\
\text { the times - strongly agree (5) to strongly disagree (1) }\end{array}$ & NA & 5 & 5 & NA & NA & NA & NA & NA & 0.904 & NA & NA & 0.548 & NA & NA & 0.139 \\
\hline
\end{tabular}

Table 1: Results of the survey. TO = Touch, SR = Speech Recognition, VA = Voice Assistant. Questions with cells marked as NA were compared between only two conditions. Significant results are marked with a grey fill.

(H1). We assume that this is due to the action button (see figure $1 \mathrm{~b}$ and figure 1e) that was featured in both conditions. Participants always looked at the screen before pressing it. However, observations show that the touch condition induced longer fixations on the screen (due to the two touch events participants had to perform) whereas for the speech recognition participants used short glances to tap the action button and acknowledged the feedback shown in figure 1 . In contrast to the touch and speech recognition, the voice assistant (Siri) offered hands and eyes-free interaction using the activation phrase "Hey Siri". Using an activation phrase instead of a button to activate the voice interaction turned out to make a significant difference when it comes to the visual attention that participants were able to spend on traffic instead of the graphical user interface. Our observations showed that participants looked at the screen only for their first interaction with Siri in order to make sure everything worked as they expected and then adopted a complete eyes-free interaction. It was interesting to see that even novices, who stated to usually not use any kind of voice assistant, adopted this behaviour quite quickly. One participant commented: "I don't use Siri as much, so I found it a little difficult at first but very intuitive". This is contrary to what Hua and $\mathrm{Ng}$ [17] find for car drivers. In their work visual feedback was recommended for users to know what commands had been given. In our case, Siri's feedback was informative enough to not create that need.

\subsection{Give Informative Feedback}

During the study, we realised that Siri gives different Feedback in German and in English, even though our technical implementation was identical. The English version would not only confirm that an obstacle was reported but also which kind. The German version did not mention the latter. Interestingly, German participants commented on this issue: "I think this is very important, if I don't look at it I would like to have the confirmation of what was created" (participant 2). We also asked participants in the interview if the confirmation helped them in general and most participants found it essential for the eye-free interaction: "Yes $100 \%$ if you are cycling at least then you know it has been logged. It is like a confirmation." (participant 3). From these opinions, we learn for future systems, that especially on the bicycle, informative confirmations are necessary for good and safe interactions. In our case, the feedback should not only confirm that the command was successfully executed but that the system actually executed the correct command.

\subsection{Keep Commands Short}

When it comes to the length and content of the commands, participants mentioned that they really liked the way the in-app speech recognition understood even short commands like "construction site" instead of full sentences. The main reason for this was that they had to remember the exact phrase for Siri to execute the command, which seemed to present a difficulty even with the commands displayed on the screen. Chang et al. [11] found that for car drivers speaking to a VUI was a lot more cognitively demanding than listening to it. Furthermore, this demand increased with the information complexity of the command that was uttered. Thus commands should be short and descriptive and the assistant should be able to resolve ambiguous commands by looking for keywords instead of complete phrases.

\subsection{Use Robust Error Handling}

In case Siri misunderstood a command and was not able to resolve the issue it responded with an error telling the participants that it had not understood the command. When this happened participants looked at the screen in order to assess the problem. This is a common behaviour for VUIs which display voice-to-text transcriptions on the screen to help users understand what the misunderstanding with the assistant was [34]. In the case of cycling, however, it might be safer to resolve such an error through a conversation with the assistant. Kim et al. [20] suggest using a simple "Can you repeat that please?" error handling method as participants in their study 
felt like this was a natural miscommunication error with only little negative impact on the interaction.

Overall, the two voice interaction techniques were more errorprone than the touch interaction. We attribute this difference mainly to the occasional error in the speech recognition as described above. However, with the ever-increasing accuracy of this technology, we expect the error rate to go down with it. For now, we argue that the increase in safety with the voice assistant outweighs the larger number of errors which can be countered by robust error handling.

\subsection{Make it safe}

When designing for interaction in motion, safety should always be a high priority. Participants explicitly talked about safety issues during our experiments. We had multiple participants comment on the fact that they would have probably severely injured themselves trying the touch screen condition while biking: "The manual thing is a no go. I mean I would die, or kill someone within like 5 minutes if I take my hands off the handlebar" (participant 5). This is especially relevant when touch interaction is still a highly used interaction technique for cycling applications. Just recently the application Bikemap [7] released a reporting feature similar to VeloCity (see figure 1c) utilising touch input. Not only touch input but also other modalities can severely impact users' safety while moving. Studies of (car) driving behaviour have shown that listening to music can influence peoples' perception and vehicular control [8]. And that phone calls during the ride can lead to reduced visual processing capabilities [31]. In regards to cyclists de Waard et al. find that mobile phone use while cycling affects cognitive demand on speed and peripheral view [14].

We know that people interact with mobile devices while moving and cyclists are no exception. Taking on the example of phone calls, Marshall et al. argue that whilst not communicating by phone at all is safer than hands-free communication, we have to assume that people will communicate while driving regardless. Given this, there is a strong argument for hands-free systems in cars [25], and we strongly believe this argument should also hold for cyclists. In this paper, we argue that we have to identify and use sensible guidelines instead of trying to enforce "stop to interact" [25] when designing interactions for cyclists.

\section{LIMITATIONS \& FUTURE WORK}

While this is the first study exploring input methods for reporting live traffic information while cycling, we recognise that our approach is prone to certain limitations. We decided to conduct the study remotely due to safety concerns. Given our results, we are convinced that participants would have been exposed to risks for which we can not take responsibility, regarding our codex as researchers as well as our local ethics board. We acknowledge that this potentially harms the ecological validity of our study. However, through the comments participants made during the experiment, we realised that participants were very often reflecting on their behaviour with regard to real cycling when evaluating the different interaction techniques. To increase future ecological validity without compromising safety we suggest looking into solutions that make use of virtual reality as shown by Löchtefeld et al. [23].
When reporting the incidents during the experiment, we embedded the commands into each video as can be seen in figure 2 to avoid that participants would forget them. This made for a fair comparison between the two voice-based and the touch-based conditions, as we have to assume that people know the commands they want to use. Furthermore, the study was conducted in two languages German and English. Therefore, all app features and surveys were translated. We did not encounter any peculiarities which made us question the validity of the results. We do believe inclusive study design improves our findings as they represent a more diverse sample and let us learn from and design for different cultures and people. Lastly, we acknowledge that the usability of the voice interaction can be highly influenced by traffic noise which we were not able to test in our setup. However, new headphones like Apple's AirPods Pro, which use a so-called transparency mode to combine noise cancellation with outside sounds, start to solve the problem of masking traffic noise and may thus be a possible way for cyclists to safely interact with a VUI. Novel technologies offer the potential to improve mobile interaction on the bicycle. Therefore, we hope that future studies and researchers will keep investigating the topic of live traffic reporting for cyclists. Many participants commented that the feedback, after logging an incident, was very informative (in the English version). Since we only used Siri's standard feedback, future studies could investigate how to optimise feedback of VUIs while cycling to learn what information cyclists need when the most. Another crucial aspect of the interaction was how the commands were formulated. Most participants preferred the short commands of the speech recognition instead of the conversational nature of Siri. Future research should thus investigate the dialogue with a voice assistant while cycling. Because, while participants preferred short commands, they liked Siri's complete answer of what was reported. We think future developments will create a need for these kinds of applications and we need to make sure that they can be interacted with in a way that is safe while cycling.

\section{CONCLUSION}

For reporting traffic incidents when driving, touch interaction is still state of the art as-is for most other mobile applications. While the availability of general-purpose navigation applications and services for cyclists is growing, we have to find ways of designing safe interaction techniques for them. Since cycling imposes a strong inhibition on touch interaction we need to find suitable interaction techniques that can be safely used on a bicycle. Voice-based interfaces on the bicycle and especially built-in voice assistants meet these requirements quite well and are safer than touch-based methods since people have to look less at the interface during the interaction. Our guidelines will help inform future designs of mobile systems for live traffic reporting for cyclists as well as for mobile applications for cyclists in general. We hope that with this paper researchers and practitioners are inspired to contribute to this topic and do so in a safe and responsible manner.

\section{ACKNOWLEDGMENTS}

We thank the participants of our study for their time. This work is part of the Volkswagen Foundation through a Lichtenberg Professorship. 


\section{REFERENCES}

[1] ADFC. 2019. ADFC Mängelmelder. Website. Retrieved August, 112020 from https://adfc-bremen.mängelmelder.de/.

[2] Robert Albrecht, Riitta Väänänen, and Tapio Lokki. 2016. Guided by Music: Pedestrian and Cyclist Navigation with Route and Beacon Guidance. Personal Ubiquitous Comput. 20, 1 (Feb. 2016), 121-145. https://doi.org/10.1007/s00779016-0906-z

[3] Tawfiq Ammari, Jofish Kaye, Janice Y. Tsai, and Frank Bentley. 2019. Music, Search, and IoT: How People (Really) Use Voice Assistants. ACM Trans. Comput.-Hum. Interact. 26, 3, Article 17 (April 2019), 28 pages. https://doi.org/10.1145/3311956

[4] Kenneth Majlund Ba h, Mads Gregers Jæger, Mikael B. Skov, and Nils Gram Thomassen. 2008. You Can Touch, but You Can't Look: Interacting with in-Vehicle Systems. In Proceedings of the SIGCHI Conference on Human Factors in Computing Systems (Florence, Italy) (CHI '08). Association for Computing Machinery, New York, NY, USA, 1139-1148. https://doi.org/10.1145/1357054.1357233

[5] Beeline. 2019. Beeline Velo. Website. Retrieved August, 112020 from https: //beeline.co/pages/beeline-velo.

[6] Hugh Beyer and Karen Holtzblatt. 1997. Contextual Design: Defining Customer Centered Systems. Morgan Kaufmann Publishers Inc., San Francisco, CA, USA.

[7] Bikemap. 2021. Bikemap. Website. Retrieved August, 112021 from https: //www.bikemap.net/de/apps/.

[8] Warren Brodsky and Micha Kizner. 2012. Exploring an alternative in-car music background designed for driver safety. Transportation Research Part F: Traffic Psychology and Behaviour 15, 2 (2012), 162 - 173. https://doi.org/10.1016/j.trf. 2011.12.001

[9] John Brooke. 1996. "SUS-A quick and dirty usability scale." Usability evaluation in industry. CRC Press. https://www.crcpress.com/product/isbn/9780748404605 ISBN: 9780748404605.

[10] Anthony Carton. 2012. Design of a Context Aware Signal Glove for Bicycle and Motorcycle Riders. In Proceedings of the 2012 ACM Conference on Ubiquitous Computing (Pittsburgh, Pennsylvania) (UbiComp '12). Association for Computing Machinery, New York, NY, USA, 635-636. https://doi.org/10.1145/2370216.2370341

[11] Chun-Cheng Chang, Jaka Sodnik, and Linda Ng Boyle. 2016. Don't Speak and Drive: Cognitive Workload of In-Vehicle Speech Interactions. In Adjunct Proceedings of the 8th International Conference on Automotive User Interfaces and Interactive Vehicular Applications (Ann Arbor, MI, USA) (AutomotiveUI '16 Adjunct). Association for Computing Machinery, New York, NY, USA, 99-104. https://doi.org/10.1145/3004323.3004351

[12] Alexandru Dancu, Zlatko Franjcic, and Morten Fjeld. 2014. Smart Flashlight: Map Navigation Using a Bike-Mounted Projector. In Proceedings of the SIGCHI Conference on Human Factors in Computing Systems (Toronto, Ontario, Canada) (CHI '14). Association for Computing Machinery, New York, NY, USA, 3627-3630. https://doi.org/10.1145/2556288.2557289

[13] Alexandru Dancu, Velko Vechev, Adviye Ayça Ünlüer, Simon Nilson, Oscar Nygren, Simon Eliasson, Jean-Elie Barjonet, Joe Marshall, and Morten Fjeld. 2015 Gesture Bike: Examining Projection Surfaces and Turn Signal Systems for Urban Cycling. In Proceedings of the 2015 International Conference on Interactive Tabletops \& Surfaces (Madeira, Portugal) (ITS '15). Association for Computing Machinery, New York, NY, USA, 151-159. https://doi.org/10.1145/2817721.2817748

[14] Dick de Waard, Paul Schepers, Wieke Ormel, and Karel Brookhuis. 2010. Mobile phone use while cycling: Incidence and effects on behaviour and safety. Ergonomics 53, 1 (2010), 30-42. https://doi.org/10.1080/00140130903381180 PMID 20069479.

[15] Debjyoti Ghosh, Can Liu, Shengdong Zhao, and Kotaro Hara. 2020. Commanding and Re-Dictation: Developing Eyes-Free Voice-Based Interaction for Editing Dictated Text. ACM Trans. Comput.-Hum. Interact. 27, 4, Article 28 (Aug. 2020), 31 pages. https://doi.org/10.1145/3390889

[16] Michael F. Goodchild and Linna Li. 2012. Assuring the quality of volunteered geographic information. Spatial Statistics 1 (2012), 110 - 120. https://doi.org/10. 1016/j.spasta.2012.03.002

[17] Zhang Hua and Wei Lieh Ng. 2010. Speech Recognition Interface Design for InVehicle System. In Proceedings of the 2nd International Conference on Automotive User Interfaces and Interactive Vehicular Applications (Pittsburgh, Pennsylvania) (AutomotiveUI '10). Association for Computing Machinery, New York, NY, USA 29-33. https://doi.org/10.1145/1969773.1969780

[18] Brianna Jean Huxtable, Carlo Ka-Ho Lai, Johnson Wen Jun Zhu, Paulina Mun-Yee Lam, Yeseul Tracy Choi, Carman Neustaedter, and Greg J. Corness. 2014. Ziklo: Bicycle Navigation through Tactile Feedback. In CHI '14 Extended Abstracts on Human Factors in Computing Systems (Toronto, Ontario, Canada) (CHI EA '14). Association for Computing Machinery, New York, NY, USA, 177-178. https: //doi.org/10.1145/2559206.2579481

[19] Shaun K. Kane, Jacob O. Wobbrock, and Ian E. Smith. 2008. Getting off the Treadmill: Evaluating Walking User Interfaces for Mobile Devices in Public Spaces. In Proceedings of the 10th International Conference on Human Computer Interaction with Mobile Devices and Services (Amsterdam, The Netherlands) (MobileHCI '08). Association for Computing Machinery, New York, NY, USA, 109-118. https: //doi.org/10.1145/1409240.1409253
[20] Jihyun Kim, Meuel Jeong, and Seul Chan Lee. 2019. "Why Did This Voice Agent Not Understand Me?": Error Recovery Strategy for in-Vehicle Voice User Interface. In Proceedings of the 11th International Conference on Automotive User Interfaces and Interactive Vehicular Applications: Adjunct Proceedings (Utrecht, Netherlands) (AutomotiveUI '19). Association for Computing Machinery, New York, NY, USA, 146-150. https://doi.org/10.1145/3349263.3351513

[21] Komoot. 2019. Komoot. Website. Retrieved August, 112020 from https://www. komoot.com/.

[22] Stephanie Krone. 2020. Die Sprecherin von @GoogleDE schreibt mir heute Spannendes: Seit Februar haben die Suchen nach Fahrradrouten via Google Maps drastisch zugenommen - um $175 \%$ ! Weltweit gab es in Corona-Zeiten ein Wachstum um 69\%. Kein Zweifel, das Fahrrad boomt! Tweet. Retrieved August, 112020 from https://twitter.com/FrauKrone/status/1285624861600231424.

[23] Markus Löchtefeld, Antonio Krüger, and Hans Gellersen. 2016. DeceptiBike: Assessing the Perception of Speed Deception in a Virtual Reality Training Bike System. In Proceedings of the 9th Nordic Conference on Human-Computer Interaction (Gothenburg, Sweden) (NordiCHI '16). Association for Computing Machinery, New York, NY, USA, Article 40, 10 pages. https://doi.org/10.1145/2971485.2971513

[24] lxian. 2019. Fuzzywuzzy_swift. Website. Retrieved July, 112021 from https: //github.com/lxian/Fuzzywuzzy_swift.

[25] Joe Marshall, Alexandru Dancu, and Florian "Floyd" Mueller. 2016. Interaction in Motion: Designing Truly Mobile Interaction. In Proceedings of the 2016 ACM Conference on Designing Interactive Systems (Brisbane, QLD, Australia) (DIS '16). Association for Computing Machinery, New York, NY, USA, 215-228. https: //doi.org/10.1145/2901790.2901844

[26] Joe Marshall and Paul Tennent. 2013. Mobile Interaction Does Not Exist. In CHI '13 Extended Abstracts on Human Factors in Computing Systems (CHI EA '13). ACM, New York, NY, USA, 2069-2078. https://doi.org/10.1145/2468356.2468725 event-place: Paris, France.

[27] Andrii Matviienko, Swamy Ananthanarayan, Shadan Sadeghian Borojeni, Yannick Feld, Wilko Heuten, and Susanne Boll. 2018. Augmenting Bicycles and Helmets with Multimodal Warnings for Children. In Proceedings of the 20th International Conference on Human-Computer Interaction with Mobile Devices and Services (Barcelona, Spain) (MobileHCI '18). Association for Computing Machinery, New York, NY, USA, Article 15, 13 pages. https://doi.org/10.1145/3229434.3229479

[28] Andrii Matviienko, Swamy Ananthanarayan, Abdallah El Ali, Wilko Heuten, and Susanne Boll. 2019. NaviBike: Comparing Unimodal Navigation Cues for Child Cyclists. In Proceedings of the 2019 CHI Conference on Human Factors in Computing Systems (Glasgow, Scotland Uk) (CHI '19). Association for Computing Machinery, New York, NY, USA, 1-12. https://doi.org/10.1145/3290605.3300850

[29] Nguyen Quang Minh. 2016. Application of "Car-Free City" and "City of Short Walks" to Living Quarters in Hanoi Towards Sustainable Mobility and Logistics. Procedia Engineering 142 (2016), 284 - 291. https://doi.org/10.1016/j.proeng.2016. 02.043 Proceeding of Sustainable Development of Civil, Urban and Transportation Engineering.

[30] Janni Nielsen, Torkil Clemmensen, and Carsten Yssing. 2002. Getting Access to What Goes on in People's Heads? Reflections on the Think-Aloud Technique. In Proceedings of the Second Nordic Conference on Human-Computer Interaction (Aarhus, Denmark) (NordiCHI '02). Association for Computing Machinery, New York, NY, USA, 101-110. https://doi.org/10.1145/572020.572033

[31] Luis Nunes and Miguel Angel Recarte. 2002. Cognitive demands of handsfree-phone conversation while driving. Transportation Research Part F: Traffic Psychology and Behaviour 5, 2 (2002), 133 - 144. https://doi.org/10.1016/S13698478(02)00012-8

[32] Martin Pielot, Benjamin Poppinga, Wilko Heuten, and Susanne Boll. 2012. Tacticycle: Supporting Exploratory Bicycle Trips. In Proceedings of the 14th International Conference on Human-Computer Interaction with Mobile Devices and Services (San Francisco, California, USA) (MobileHCI '12). Association for Computing Machinery, New York, NY, USA, 369-378. https://doi.org/10.1145/2371574.2371631

[33] Benjamin Poppinga, Martin Pielot, and Susanne Boll. 2009. Tacticycle: A Tactile Display for Supporting Tourists on a Bicycle Trip. In Proceedings of the 11th International Conference on Human-Computer Interaction with Mobile Devices and Services (Bonn, Germany) (MobileHCI'09). Association for Computing Machinery, New York, NY, USA, Article 41, 4 pages. https://doi.org/10.1145/1613858.1613911

[34] Martin Porcheron, Joel E. Fischer, Stuart Reeves, and Sarah Sharples. 2018. Voice Interfaces in Everyday Life. In Proceedings of the 2018 CHI Conference on Human Factors in Computing Systems (Montreal QC, Canada) (CHI '18). Association for Computing Machinery, New York, NY, USA, 1-12. https://doi.org/10.1145/ 3173574.3174214

[35] Felix Richter. 2018. The Global Rise of Bike-Sharing. Statista (2018). Retrieved September 8, 2020 from https://www.statista.com/chart/13483/bike-sharingprograms/

[36] Felix Richter. 2020. Infographic: COVID-19 Pandemic Fuels Bicycle Boom. https://www.statista.com/chart/21794/year-over-year-change-inbicycle-sales-in-the-us/ Library Catalog: www.statista.com.

[37] Judy Robertson and Maurits Kaptein. 2016. Modern Statistical Methods for HCI (1st ed.). Springer Publishing Company, Incorporated, Switzerland. 
[38] Duncan Rowland, Martin Flintham, Leif Oppermann, Joe Marshall, Alan Chamberlain, Boriana Koleva, Steve Benford, and Citlali Perez. 2009. Ubikequitous Computing: Designing Interactive Experiences for Cyclists. In Proceedings of the 11th International Conference on Human-Computer Interaction with Mobile Devices and Services (Bonn, Germany) (MobileHCI '09). Association for Computing Machinery, New York, NY, USA, Article 21, 11 pages. https://doi.org/10.1145/1613858.1613886

[39] Gian-Luca Savino, Laura Meyer, Eve Emily Sophie Schade, Thora Tenbrink, and Johannes Schöning. 2020. Point Me In the Right Direction: Understanding User Behaviour with As-The-Crow-Flies Navigation (MobileHCI '20). Association for Computing Machinery, New York, NY, USA. https://doi.org/10.1145/3379503. 3403539

[40] Paul Schepers and Karin Klein Wolt. 2012. Single-Bicycle Crash Types and Characteristics. Cycling Research International 2 (01 2012), 119 - 135.

[41] SmartHalo. 2019. SmartHalo 2. Website. Retrieved August, 112020 from https://www.smarthalo.bike/.

[42] Haska Steltenpohl and Anders Bouwer. 2013. Vibrobelt: Tactile Navigation Support for Cyclists. In Proceedings of the 2013 International Conference on Intelligent User Interfaces (Santa Monica, California, USA) (IUI '13). Association for Computing Machinery, New York, NY, USA, 417-426. https://doi.org/10.1145/2449396. 2449450

[43] Strava. 2019. Strava. Website. Retrieved August, 112020 from https://www. strava.com.

[44] Delphine Szymczak, Kirsten Rassmus-Gröhn, Charlotte Magnusson, and PerOlof Hedvall. 2012. A Real-World Study of an Audio-Tactile Tourist Guide. In
Proceedings of the 14th International Conference on Human-Computer Interaction with Mobile Devices and Services (San Francisco, California, USA) (MobileHCI '12). Association for Computing Machinery, New York, NY, USA, 335-344. https: //doi.org/10.1145/2371574.2371627

[45] D. van Lierop, J. Soemers, L. Hoeke, G. Liu, Z. Chen, D. Ettema, and J. Kruijf. 2020. Wayfinding for cycle highways: Assessing e-bike users' experiences with wayfinding along a cycle highway in the Netherlands. Fournal of Transport Geography 88 (2020), 102827. https://doi.org/10.1016/j.jtrangeo.2020.102827

[46] Velko Vechev, Alexandru Dancu, Simon T. Perrault, Quentin Roy, Morten Fjeld, and Shengdong Zhao. 2018. Movespace: On-Body Athletic Interaction for Running and Cycling. In Proceedings of the 2018 International Conference on Advanced Visual Interfaces (Castiglione della Pescaia, Grosseto, Italy) (AVI '18). Association for Computing Machinery, New York, NY, USA, Article 28, 9 pages. https://doi.org/10.1145/3206505.3206527

[47] Waze. 2019. Waze. Website. Retrieved August, 112020 from https://www.waze. com/.

[48] Pawel W. Woźniak, Lex Dekker, Francisco Kiss, Ella Velner, Andrea Kuijt, and Stella F. Donker. 2020. Brotate and Tribike: Designing Smartphone Control for Cycling (MobileHCI '20). Association for Computing Machinery, New York, NY, USA. https://doi.org/10.1145/3379503.3405660

[49] YouGov Italy. 2020. Italy: bicycle use intentions post-lockdown 2020. https://www.statista.com/statistics/1123452/future-bicycle-general-usepatterns-after-the-lockdown-in-italy/ Library Catalog: www.statista.com. 